\title{
BRAND SENSUALITY \& BRAND AFFECT: OS SEUS ANTECEDENTES E CONSEQUENTES
}

\author{
BRAND SENSUALITY AND BRAND AFFECT: ITS \\ ANTECEDENTS AND CONSEQUENCES
}

\section{RESUMO}

Os afetos são relevantes para que os consumidores possam desenvolver relações próximas e especiais com as marcas. Nesse âmbito, esta pesquisa tem como objetivo principal compreender quais os antecedentes e consequentes da "Brand Sensuality" e do "Brand Affect" e testar o efeito moderador do género. Por meio da realização de um inquérito, obtiveram-se 234 respostas válidas (do sexo feminino e do sexo masculino). De forma a testar as 19 hipóteses de investigação propostas ao longo do modelo conceptual, recorreu-se ao modelo das equações estruturais. Este trabalho de investigação revelou o impacto positivo da "Brand Sensuality" sobre o "Brand Affect", trazendo, assim, contributos essenciais para o universo das marcas. Os resultados devem ainda ser um estímulo para a investigação na área do marketing e do Branding, uma vez que, por meio do desenvolvimento dessa investigação, clarifica-se, em nível prático, o conceito de "Brand Sensuality", muitas vezes, confundido e referenciado como "Brand Sense". Por outro lado, desvendam-se alguns dos seus antecedentes e consequentes e consolidam-se os conceitos de "Brand Affect" e "Brand Sensuality".

Carlos Daniel Vaz Nunes carlos-3@live.com.pt Mestre em Marketing pela Faculdade de Economia da Universidade de CoimbraPortugal.

\section{Arnaldo Coelho} coelho1963@hotmail.com PHD em Gestão. Professor e coordenador dos cursos de doutoramento e MBA da Universidade de CoimbraPortugal.

Cristela Maia Bairrada cristela.bairrada@uc.pt PHD em Gestão. Professora na Faculdade de Economia da Universidade de Coimbra.
Palavras-chave: Sensualidade da Marca. Afeto à Marca. Antecedentes. Consequentes. Gênero.

\begin{abstract}
Affections are relevant so that consumers can develop close and special relationships with brands. In this context, this research aims to understand the antecedents and consequences of "Brand Sensuality" and "Brand Affect" and to test the moderating effect of gender. Through a survey, 234 valid answers (female and male) were obtained. In order to test the 19 research hypotheses proposed along the conceptual model, we resorted to the structural equations model. This research work revealed the positive impact of "Brand Sensuality" on "Brand Affect", thus bringing essential contributions to the universe of brands. The results should also be
\end{abstract}


a stimulus for research in marketing and branding, since, through the development of this research, the concept of "Brand Sensuality", often confused and referred to as "Brand Sense", is clarified in practical terms. On the other hand, some of its antecedents and consequences are unveiled and the concepts of "Brand Affect" and "Brand Sensuality" are consolidated.

Keywords: Brand Sensuality. Brand Affect. Antecedents. Consequences. Gender.

\section{INTRODUÇÃO}

A presente investigação pretende clarificar o papel dos afetos e da sensualidade nas relações entre consumidores e marcas, explorando ambos os conceitos e identificando os seus antecedentes e consequentes. A importância desta investigação assenta na possibilidade de auxiliar empresas a focarem-se mais nas relações que estabelecem com os seus consumidores, procurando responder às suas necessidades. Tendo como referência o espetro relacional e sabendo que os consumidores podem atribuir caraterísticas humanas às marcas (AAKER, 1997; FOURNIER, 1998), o afeto à marca e sensualidade da marca são conceitos de importância não só porque permitem uma melhor compreensão do comportamento do consumidor, mas também porque esses sentimentos podem solidificar os relacionamentos entre consumidores e marcas (SARKAR; PONNAM, 2012).

Nesse contexto, compreender o consumidor parece ser a chave para a criação de um posicionamento capaz de levar determinada marca a atingir o sucesso. Assim como nos relacionamentos pessoais, inúmeros estudos revelam que os consumidores desenvolvem sentimentos com as marcas (CARROLL; AHUVIA, 2006; CHO; FIORE; RUSSELL, 2015; EREVELLES, 1998; ROBERTS, 2005; VALDEZ; MEHRABIAN, 1994). Consequentemente, investigadores do marketing tentam perceber a diversidade de construtos que decorrem dessa relação entre marcas e consumidores.
Dada a importância das marcas no contexto do marketing, têm aumentado os estudos sobre essa temática, tendo surgido inúmeros conceitos que giram em torno do conceito de marca. Chega-se, assim, à conclusão de que as marcas, para conseguirem o coração e a lealdade verdadeira dos clientes, precisam superar muito mais do que o lado racional. É preciso alcançar ligações genuinamente emocionais, pois, somente dessa forma, os sentimentos desenvolvidos entre marcas e consumidores poderão perdurar no tempo (SUNG; KIM, 2010).

Destaca-se que a marca escolhida como objeto de estudo para este trabalho de investigação foi a marca Nespresso. A escolha dessa marca deveu-se ao fato de existirem poucas abordagens que evidenciam essa relação da marca Nespresso com o comportamento do consumidor. A marca Nespresso surgiu em 1986, tendo como principal objetivo o de reinventar e revolucionar o modo como milhares de consumidores desfrutavam do seu café (BREM; MAIER; WIMSCHNEIDER, 2016). O leque de experiências aromáticas faz que a Nespresso atinja a mente e o coração dos consumidores

O principal objetivo dessa investigação será realizar um estudo empírico no sentido de explicar os antecedentes e consequentes do "Brand Affect" e "Brand Sensuality". Com as mudanças constantes dos perfis de consumo e comportamentos do consumidor, as marcas também acompanham uma metamorfose simultânea, sendo equiparadas ao ser humano. A perceção dessas variáveis poderá acarretar mais-valias ao nível empresarial uma vez que permitirá o desenvolvimento de novas estratégias que irão enriquecer a estrutura de fidelização de clientes.

Apesar de a "Brand Sensuality" se encontrar em uma fase embrionária, existindo muito trabalho de investigação para uma melhor compreensão desse novo construto do marketing, esta pesquisa representa uma análise capaz de melhorar o entendimento do comportamento do consumidor. Nesse âmbito, serão analisados vários aspetos de natureza mais simbólica na determinação da sensualidade e afeto à marca, 
bem como será testada a universalidade de efeitos observados noutras investigações referentes a essas variáveis.

\section{QUADRO CONCEITUAL E HIPÓTESES DE INVESTIGAÇÃO}

\subsection{QUADRO CONCEITUAL}

Se por um lado verificamos que o conceito de "Brand Affect" já se encontra bastante explorado na literatura acadêmica, por outro, verificamos que, quando falamos de "Brand Sensuality", estamos perante um tema recente e, por isso, pouco claro na literatura do marketing.

\subsubsection{O conceito de Afeto}

Segundo Chaudhuri e Holbrook (2001), $\mathrm{o}$ afeto à marca decorre de um relacionamento próximo com a marca, ou seja, corresponde à resposta emocional positiva dos consumidores em relação a uma marca.

Bagozzi, Gopinath e Nyer, (1999) referem que o afeto pode ser visto como um processo complexo e de difícil compreensão, sendo considerado, de forma generalizada, como uma categoria dos sentimentos. Além disso, diferenciam-se três tipos de formas afetivas: emoções, humor e atitudes. $\mathrm{O}$ afeto é tido assim como um conceito mais geral e refere-se a um sentimento consciente; contudo, não reflexivo e cognitivo, enquanto na emoção os processos de avaliação podem traduzir-se por conscientes ou inconscientes (RUSSELL, 2003; RUSSELL; BARRETT, 1999).

\subsubsection{Conceito de Marca}

Segundo Chernatony e Riley (1998), o conceito de marca delimita-se segundo 12 premissas relevantes. Assim, a marca pode ser vista como um instrumento legal, um logótipo, uma empresa, uma estenografia, um redutor de risco, um sistema de identificação, uma imagem na mente do consumidor, um sistema de valor, algo com personalidade, um elemento de rela- cionamento, um acréscimo de valor e, por último, algo que evolui com o tempo. Conclui-se, assim, que existem múltiplas definições para o conceito de marca (CHERNATONY; RILEY, 1998). Segundo Keller (1993), um consumidor armazena na sua memória um conjunto de aspetos sobre determinada marca, podendo, desse modo, assumir o papel de benefícios (valor e significado que os consumidores colocam às marcas); consciência (envolve a identificação da categoria e das necessidades que a marca satisfaz); atributos (aspetos relacionados com o desemprenho e a personalidade); imagens (informação visual que está associada às marcas); pensamentos (respostas cognitivas); sentimentos (respostas afetivas); atitudes (avaliação que o consumidor faz à determinada marca) e, por último, experiências (relacionadas com o comportamento de consumo e compra).

\subsubsection{Brand Affect}

Em primeiro lugar, importa referir que o "Brand Affect" pode ser considerado como uma avaliação positiva ou negativa, que um consumidor associa a uma determinada marca (MATZLER; BIDMON; GRABNER-KRÄUTER, 2006). Em segundo lugar, sempre que ocorrer uma visualização na mente do consumidor de uma marca, surge uma recordação afetiva associada a ela (SUNG; KIM, 2010; ZAJONC, 1980). Essas recordações acarretam sempre consigo lembranças afetivas tanto positivas como negativas (TELLEGEN; WATSON; CLARK, 1999). Por um lado, o afeto positivo pela marca vai influenciar os consumidores a nutrirem bons sentimentos e recordações, que irá evoluir de forma progressiva e gradual na mente deles (CHI; YEH; CHIOU, 2009). Todavia, o afeto negativo pela marca irá induzir à insatisfação e, consequentemente, ao passa-palavra negativo (CHI; YEH; CHIOU, 2009).

As respostas afetivas de um consumidor perante determinada marca revelam-se de extrema importância, ou seja, o afeto sentido por uma marca é um bom elo entre consumidor e marca (CHAUDHURI; HOLBROOK, 2001). Dessa 
forma, as marcas têm um papel fundamental em estimular o consumidor de forma a que se sinta alegre, feliz ou acarinhado (MATZLER; BIDMON; GRABNER-KRÄUTER, 2006).

\subsubsection{Brand Sensuality}

No mundo contemporâneo das marcas, luta-se, desenfreadamente, pela satisfação e fidelização dos clientes, tornando, assim, a sensualidade ainda mais relevante e importante como um item diferenciador para despertar os sentidos de homens e mulheres (AMOR et al., 2013).

Segundo Roberts (2005), as marcas têm vindo, cada vez mais, a utilizar a sensualidade nas suas estratégias de comunicação, apelando aos sentidos, inspiração e sedução. Aspetos como beleza, sensualidade e erotismo são transformados em um processo geral de materialização do corpo, ou seja, representam-se como armas privilegiadas para captar o foco do consumidor (LIPOVETSKY, 2000; SAMARÃO, 2007).

Lipovetsky (2000), Gonçalves e Nishida (2009) referem que ainda é recorrente em algumas campanhas a construção da imagem da mulher vinculada à sensualidade e ao erotismo de forma a produzir anúncios baseados na cognição e no desejo sensual.

Se antes a sensualidade feminina era vista somente no campo da conquista, hoje se pode dizer que ela se apresenta para seduzir o homem, mas também para inspirar a mulher (FERNANDES, 2010; SOUZA; LEÃO, 2013; TEIXEIRA, 2009). Na maior parte das campanhas publicitárias, a sensualidade está presente de forma camuflada, aprestando-se aos consumidores como uma projeção de si mesmos, ou seja, essas campanhas publicitárias têm como objetivo lembrar aos consumidores, tanto feminino como masculino, sobre quem são e o que desejam ser (TEIXEIRA, 2009). Esse papel da publicidade é um dos fatores relevantes para a formulação de gênero e identidade (SABAT, 2001; TEIXEIRA, 2009). Esses aspetos são, indiscutivelmente, construídos nas relações históricas, sociais e culturais, permitindo, as- sim, descrever a diferença entre o feminino e o masculino (SABAT, 2001; TEIXEIRA, 2009).

A sensualidade está fortemente vinculada aos padrões estéticos; por isso, as marcas trabalham as campanhas publicitárias com imagens de corpos esbeltos e magros dotados de uma perfeição apurada e cuidada, muitas vezes por meio do auxílio de softwares que editam, cuidadosamente, suas imagens (SOUZA; LEÃO, 2013), ultrapassando-se os limites da beleza humana (FERNANDES, 2010). Esse corpo feminino perfeito é fabricado e tratado pelas marcas como um produto de consumo, modificado e representado, muitas vezes, de modo preconceituoso e sexista (SAMARÃO, 2007). Dessa forma, a aparência e o corpo assumem uma grande importância nas relações sociais dos consumidores, ou seja, fruto da supervalorização do aspeto estético, principalmente, ligado ao universo da moda e do consumo (TEIXEIRA, 2009).

Gould (1992b) evidencia que o sex appeal pode ser encontrado na visão, audição e nos elementos verbais que as marcas utilizam em suas campanhas publicitárias. Segundo Roberts (2005), uma composição musical, uma estimulação olfativa, uma variedade de texturas, as experiências visuais, o logotipo e até a embalagem de determinado produto conduzem a associações agradáveis, produzindo, assim, no consumidor o desejo sensual. Quando as marcas conseguem apelar aos sentidos dos clientes, podem encontrar o caminho que leva a seus corações. Logo um consumidor molda sua perceção e sentimentos por uma marca por meio das experiências que captam os efeitos cognitivos, sensoriais e emocionais; dessa forma, Roberts (2005) divide essas perceções em três dimensões relevantes: mistério, intimidade e sensualidade.

\subsection{HIPÓTESES DE INVESTIGAÇÃO}

\subsubsection{Brand Sensuality e Brand Affect}

O "Brand Sense" e "Brand Sensuality" tratam-se de construtos díspares. Assim, aferimos que "Brand Sense" designa-se como 
o marketing e todo o conjunto dos sentidos humanos que afetam as perceções, os julgamentos e os comportamentos dos consumidores (KRISHNA, 2012; LINDSTROM, 2007), enquanto a "Brand Sensuality" absorve parte do "Brand Sense", uma vez que experiências multissensoriais induzem, na mente do consumidor, o apelo sensual por meio do sex appel (GOULD, 1992b; ROBERTS, 2005). Todavia, a sensualidade da marca é, sem dúvida, um construto mais abrangente, ou seja, esse construto desperta ao consumidor prazeres e apelos sensuais por meio do contato com determinada marca, criando um sentimento de excitação, levando o consumidor a ficar fora de si (FERNANDES, 2010; GONÇALVES; NISHIDA, 2009; LIPOVETSKY, 2000; PEREIRA; COELHO; BAIRRADA, 2016; SABAT, 2001; SAMARÃO, 2007; SOUZA; LEÃO, 2013; TEIXEIRA, 2009; VERÍSSIMO, 2005), podendo até criar afetos por determinada marca (CHO; FIORE; RUSSELL, 2015).

Assim, propõe-se que:

H1: A "Brand Sensuality" tem um impacto positivo sobre o "Brand Affect".

\subsubsection{Antecedentes do Brand Sensuality e Brand Affect}

\subsubsection{Brand Experience}

O "Brand Experience" carateriza-se como algo subjetivo, constituído por respostas internas do consumidor (sensações, sentimentos e cognições) e respostas comportamentais (BRAKUS; SCHMITT; ZARANTONELLO, 2009). Segundo Schmitt (1999), o marketing afetivo apela para os sentimentos e as emoções intrínsecas, com o objetivo de suscitar experiências afetivas que vão desde sensações levianas até sensações fortes, como, por exemplo, a alegria e o orgulho relativos a uma marca.

De acordo com Rodrigues (2014), assume-se que a sensualidade da marca desempenha um papel importante na ligação e na interação holística entre marcas e consumidores que pretendem alcançar benefícios sensoriais, sedutores e experiências impares no processo de criação de valor entre marca e consumidor. Segundo Hoch (2002), os aspetos sedutores provenientes de determinada experiência levam os consumidores a envolverem-se e a desenvolverem uma sensação ímpar e sem igual com essa marca.

Assim, propõe-se que:

H2: O "Brand Experience" tem um impacto positivo sobre o "Brand Affect".

H3: O "Brand Experience" tem um impacto positivo sobre a "Brand Sensuality".

\subsubsection{Hedonic product}

Segundo Dhar e Wertenbroch (2000), as escolhas dos consumidores resultam de pressupostos relacionados com questões hedônicas e utilitárias.

Carpenter e Fairhurst (2005) afirmam que os consumidores tendem a pagar um preço mais alto por produtos de luxo e procuram, sobretudo, valores utilitários e hedônicos por meio da experiência associada na compra, isto é, recorrem aos bens de luxo para corresponder às necessidades com base nas suas expectativas, significado social, bem como a qualidade associada aos produtos.

Uma vez que os produtos hedônicos estão diretamente associados a emoções e prazer, é expetável que o consumo desses produtos desperte fortes respostas emocionais e afetivas (CARROLL; AHUVIA, 2006).

Dessa forma, propõe-se que:

H4: O "Hedonic product" tem um impacto positivo sobre o "Brand Affect".

H5: O "Hedonic product" tem um impacto positivo sobre a "Brand Sensuality".

\subsubsection{Brand Identity Expressiveness}

A "Brand Identity Expressivenes" é definida como a capacidade na qual determinada marca constrói e simboliza a identidade do indivíduo, bem como uma identidade social perante terceiros (XIE; BATRA; PENG, 2015). Os consumidores podem instituir caraterísticas humanas nas marcas e pensarem nelas como 
se fossem celebridades, figuras importantes ou históricas (AAKER, 1997; PLUMMER, 2000; ROOK, 1985). Assim, o valor de uma marca é cocriado por relacionamentos afetivos entre marcas e consumidores, resultado de experiências vividas com as marcas de forma direta ou indireta (GOULD, 1992b).

Assim, propõe-se que:

H6: O "Brand Identity Expressivenes" tem um impacto positivo sobre o "Brand Affect".

H7: O "Brand Identity Expressivenes" tem um impacto positivo sobre a "Brand Sensuality".

\subsubsection{Brand Trust}

A confiança é o ingrediente-chave para o desenvolvimento de relações com as marcas (CARROLL; AHUVIA, 2006; MCALEXANDER; SCHOUTEN; KOENIG, 2002; MORGAN; HUNT, 1994). Schoorman, Mayer e Davis (2007) referem que, no momento em que as emoções estão a ser vivenciadas, elas podem levar o indivíduo a atualizar as perceções prévias nas dimensões da confiabilidade e confiança de tal forma que, mesmo depois das emoções desaparecerem, o efeito na avaliação cognitiva permanece, ou seja, as emoções têm um efeito primário sobre a confiança, evoluindo, subsequentemente, para uma resposta cognitiva. Segundo Herskovitz e Crystal (2010), quando uma marca permanece fiel aos seus valores, esta consegue crescer em longo prazo e adaptar-se a situações de mudança. Cumprindo-se essas premissas as marcas evocam fortes respostas emocionais nos clientes, incluindo, lealdade, confiança e devoção.

Dessa forma, propõe-se que:

H8: O "Brand Trust" tem um impacto positivo sobre o "Brand Affect".

H9: O "Brand Trust" tem um impacto positivo sobre a "Brand Sensuality".

\subsubsection{Brand Intimacy}

A intimidade designa os sentimentos de proximidade, conexão e vínculo nos relacionamentos amorosos. A sensualidade da marca está, intrinsecamente, associada à intimidade, sendo recorrente a literatura descrever a importância das experiências íntimas entre marcas e consumidores para desencadear perceções associadas ao sex appel (GONÇALVES; NISHIDA, 2009; GOULD, 1992b; ROBERTS, 2005; SAMARÃO, 2007).

Assim, propõe-se que:

H10: O "Brand Intimacy" tem um impacto positivo sobre o "Brand Affect".

H11: O "Brand Intimacy" tem um impacto positivo sobre a "Brand Sensuality".

\subsubsection{Consequências do Brand Sensuali- ty e Brand Affect}

\subsubsection{Brand Loyalty}

Segundo Shoemaker e Lewis 1999, existem duas premissas fundamentais para atingir a lealdade, uma referente à ligação emocional/ afetiva com a marca e outra relacionada com a repetição da compra de determinada marca.

As marcas desempenham um papel fundamental na formação de experiências multissensoriais, incorporando associações que podem atrair o consumidor por meio da estimulação dos sentidos, das perceções de afetos e dos desejos sensuais, suscitados no momento de decisão de compra, sendo reforçada a lealdade e a perceção emocional entre marca e consumidor (CHAUDHURI; HOLBROOK, 2001; CHO; FIORE; RUSSELL, 2015; DICK; BASU, 1994; FENKO; SCHIFFERSTEIN; HEKKERT, 2010; FIORE; KIM, 2007; FIORE; YAH; YOH, 2000; LIN; LEE, 2012; ROBERTS, 2005; WORTHINGTON; RUSSELL-BENNETT; HÄRTEL, 2009).

Dessa forma, propõe-se que:

H11: O "Brand Affect" tem um impacto positivo sobre o "Brand Loyalty".

H12: O "Brand Sensuality" tem um impacto positivo sobre a "Brand Loyalty".

\subsubsection{WOM+}

Segundo Carroll e Ahuvia (2006), o con- 
ceito de WOM está relacionado com o fato de o consumidor falar bem e recomendar determinada marca a um coletivo abrangente ou a singulares, como, por exemplo, a amigos. Segundo Batra, Ahuvia e Bagozzi (2012) os consumidores que nutrem afetos por uma marca estão mais sujeitos a falar dela.

Fog, Budtz e Yakaboylu (2005) acrescentam que uma história bem contada tem um enorme potencial indo além do próprio branding. Roberts (2005), Sametz e Maydoney (2003) afirmam que as histórias nunca devem tornar-se óbvias nem muito claras, ou seja, as expetativas geradas serão tanto maiores quando maior for o mistério. Assim, propomos que o mistério conduz ao word-of-mouth, existindo uma conexão entre marca e consumidor. Assim, propõe-se que:

H14: O "Brand Affect" tem um impacto positivo sobre o "WOM+".

H15: O "Brand Sensuality" tem um impacto positivo sobre a "WOM+".

\subsubsection{Brand Love}

O amor está ligado a emoções positivas. No contexto das marcas, espera-se que o afeto positivo tenha impacto no nível da duração do relacionamento (BAIRRADA, 2015; BATRA; AHUVIA; BAGOZZI, 2012). Roberts (2005) afirma que a sensualidade é uma das variáveis fundamentais para despertar o sentimento de amor pela marca.

Assim, propõe-se que:

H16: O "Brand Affect" tem um impacto positivo sobre o "Brand Love".

H17: O "Brand Sensuality" tem um impacto positivo sobre a "Brand Love".

\subsubsection{Price Premium}

A sensibilidade ao preço descreve a perceção de determinado consumidor às alterações de níveis de preços, ou seja, cada consumidor tem um intervalo de aceitação de preços praticados. Desse modo, propõ-se que o afeto e a sensualidade têm uma relação positiva com a disponibilidade de pagar mais por determinada marca. Ou seja, os valores emocionais e atrações sensuais pela marca sobrepõem-se a qualquer nível de preço (BAIRRADA, 2015; FEYBESSE; NETO; HATFIELD, 2011).

Dessa forma, propõe-se que:

H18: O "Brand Affect" tem um impacto positivo sobre o "Price Premium".

H19: O "Brand Sensuality" tem um impacto positivo sobre a "Price Premium".

A figura 1 apresenta, graficamente, o modelo conceitual de investigação e as relações sugeridas entre as variáveis consideradas na presente pesquisa.

Figura 1 - Representação gráfica do modelo conceitual da investigação- Brand Sensuality \& Brand Affect: os seus antecedentes e consequentes

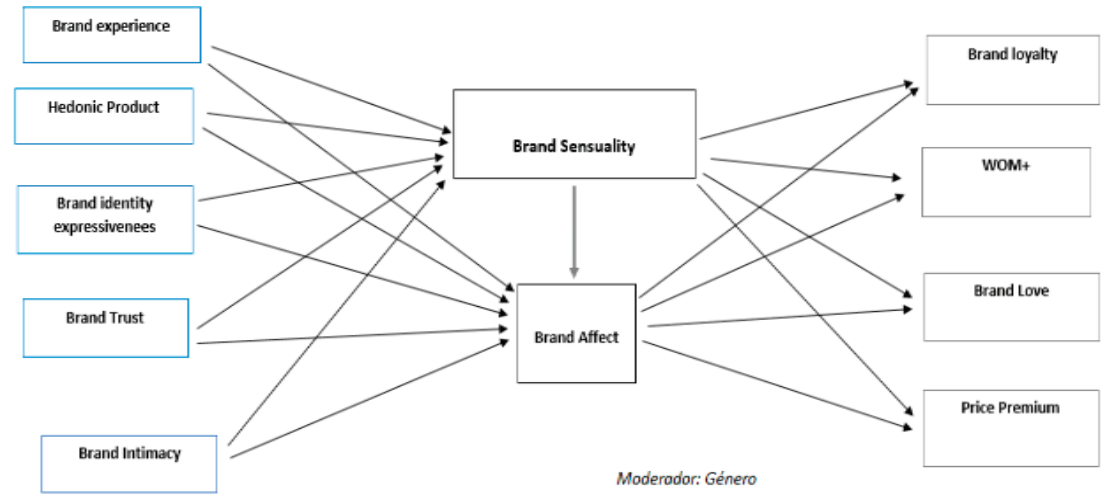

Fonte: elaboração própria. 


\section{METODOLOGIA}

\subsection{RECOLHA DE DADOS}

A técnica de recolha de dados selecionada passou, inicialmente, pela elaboração de um questionário online, por meio da plataforma Google Forms. Esse mesmo questionário foi partilhado nas diversas plataformas digitais para recolher o máximo de respostas possíveis. A pesquisa foi direcionada apenas aos consumidores de Nespresso, não sendo colocada qualquer tipo de restrição à nacionalidade dos inquiridos. Todas as questões do inquérito tiveram um formato de escolha múltipla. A mensuração da opinião dos inquiridos foi feita por meio da Escala de Likert de 7 pontos, em que o 1 representava a resposta "Discordo Totalmente" e o 7 significava "Concordo Totalmente".

Relativamente aos dados recolhidos, evidencia-se que, no total dos 234 respondentes,
$58,1 \%$ (136 indivíduos) eram do sexo feminino, enquanto $41,9 \%$ (98 indivíduos) eram do sexo masculino. Observamos que 87 indivíduos $(37,18 \%)$ tinham uma idade compreendida entre os 20 e 29 anos. A média de idade dos 234 inquiridos da amostragem é de, aproximadamente, 35 anos de idade. No que se refere ao modelo de medidas, constataram-se estatísticas e índices reveladores de um bom ajustamento: $\chi 2 / \mathrm{gl}=1,996, \mathrm{IFI}=0,35, \mathrm{TLI}=0,929, \mathrm{CFI}=$ 0,935 , RMSEA $=0,065$. Para todas as variáveis latentes, quer os valores da fiabilidade compósita (CR), quer os valores da variância extraída média (AVE), estão, respetivamente, acima dos valores recomendados de 0.7 e 0.5 . Verificamos que todos os construtos no modelo conceptual proposto respeitam o critério estipulado por Hair et al. (2014), como comprova a tabela 1, concluindo-se que os indicadores representam bem a variável que pretendem medir (LISBOA; AUGUSTO; FERREIRA, 2012).

Tabela 1 - Desvio-padrão; diagonal a negrito - Alpha de Cronbach; C.R. - Fiabilidade compósita; AVE Variância média extraída

\begin{tabular}{|c|c|c|c|c|c|c|c|c|c|c|c|c|c|c|}
\hline Variáveis & DP & BS & $B A$ & Exp & $H_{-} P$ & Id_Ex & Tst & Incy & Loy & WOM & Love & $P_{-} P$ & AVE & CR \\
\hline $\begin{array}{l}\text { Brand Sensuality } \\
\text { (BS) }\end{array}$ & 1,658 & 0,902 & & & & & & & & & & & 0,720 & 0,937 \\
\hline Brand Affect (BA) & 1,843 & 0,852 & 0,973 & & & & & & & & & & 0,901 & 0,973 \\
\hline $\begin{array}{l}\text { Brand Experience } \\
\text { (Exp) }\end{array}$ & 1,675 & 0,85 & 0,839 & 0,974 & & & & & & & & & - & - \\
\hline $\begin{array}{l}\text { Hedonic Product } \\
\text { (H_P) }\end{array}$ & 1,854 & 0,79 & 0,855 & 0,833 & 0,959 & & & & & & & & 0,829 & 0,960 \\
\hline $\begin{array}{l}\text { Brand Identity } \\
\text { expressivess } \\
\text { (Id_Ex) }\end{array}$ & 1,855 & 0,641 & 0,571 & 0,758 & 0,614 & 0,970 & & & & & & & 0,920 & 0,972 \\
\hline Brand Trust (Tst) & 1,687 & 0,628 & 0,704 & 0,687 & 0,763 & 0,474 & 0,967 & & & & & & 0,883 & 0,968 \\
\hline $\begin{array}{l}\text { Brand Intimacy } \\
\text { (Incy) }\end{array}$ & 1,825 & 0,637 & 0,618 & 0,723 & 0,686 & 0,642 & 0,715 & 0,974 & & & & & 0,928 & 0,975 \\
\hline Brand Loyalty (Loy) & 1,941 & 0,636 & 0,677 & 0,699 & 0,721 & 0,579 & 0,762 & 0,706 & 0,944 & & & & 0,813 & 0,945 \\
\hline wom & 1,966 & 0,631 & 0,692 & 0,682 & 0,754 & 0,503 & 0,758 & 0,673 & 0,888 & 0,964 & & & 0,873 & 0,965 \\
\hline Brand Love (Love) & 1,846 & 0,66 & 0,657 & 0,745 & 0,721 & 0,653 & 0,654 & 0,733 & 0,839 & 0,788 & 0,978 & & 0,884 & 0,979 \\
\hline Price Primium(P_P) & 1,760 & 0,503 & 0,566 & 0,641 & 0,596 & 0,549 & 0,533 & 0,538 & 0,717 & 0,674 & 0,684 & 0,946 & 0,814 & 0,946 \\
\hline
\end{tabular}

Fonte: elaboração própria

\section{ANÁLISE E DISCUSSÃO DE RESULTADOS}

\subsection{RESULTADOS}

Por fim, o modelo estrutural apresenta estatísticas e índices de um ajustamento ade- quado: $\chi 2 / \mathrm{gl}=2,354, \mathrm{IFI}=0,910, \mathrm{TLI}=0,904$, $\mathrm{CFI}=0,910, \mathrm{RMSEA}=0,076$. 
Tabela 2- Resultado do Teste de Hipóteses

\begin{tabular}{|c|c|c|c|c|c|c|c|c|c|}
\hline \multirow{3}{*}{ Hipóteses } & & & \multirow{2}{*}{\multicolumn{2}{|c|}{$\begin{array}{l}\text { Geral } \\
N=234\end{array}$}} & \multirow{2}{*}{\multicolumn{3}{|c|}{$\begin{array}{l}\mathrm{P}=136 \\
\mathrm{~N}=136\end{array}$}} & \multirow{2}{*}{\multicolumn{2}{|c|}{$\begin{array}{l}\hat{N}=98\end{array}$}} \\
\hline & & & & & & & & & \\
\hline & & & $\mathrm{H}$ & SRW & $\mathrm{P}$ & SRW & $\mathrm{P}$ & SRW & $\mathrm{P}$ \\
\hline Brand Sensuality & $\rightarrow$ & Brand Affect & H1 & 0,388 & $* * *$ & 0,514 & $* * *$ & 0,223 & NS \\
\hline Brand Experience & $\rightarrow$ & Brand Affect & $\mathrm{H} 2$ & 0,198 & $*$ & $-0,127$ & NS & 0,367 & $* *$ \\
\hline Brand Experience & $\rightarrow$ & Brand Sensuality & $\mathrm{H} 3$ & 0,659 & $* * *$ & 0,79 & $* * *$ & 0,5 & $* * *$ \\
\hline Hedonic Product & $\rightarrow$ & Brand Affect & $\mathrm{H} 4$ & 0,352 & $* * *$ & 0,555 & $* * *$ & 0,312 & $* *$ \\
\hline Hedonic Product & $\rightarrow$ & Brand Sensuality & H5 & 0,152 & NS & $-0,003$ & NS & 0,362 & $* * *$ \\
\hline $\begin{array}{l}\text { Brand identity } \\
\text { expressivenees }\end{array}$ & $\rightarrow$ & Brand Affect & H6 & $-0,063$ & NS & 0,072 & NS & $-0,195$ & $* *$ \\
\hline $\begin{array}{l}\text { Brand identity } \\
\text { expressivenees }\end{array}$ & $\rightarrow$ & Brand Sensuality & $\mathrm{H} 7$ & 0,022 & NS & $-0,086$ & NS & 0,172 & $*$ \\
\hline Brand Trust & $\rightarrow$ & Brand Affect & $\mathrm{H} 8$ & 0,145 & $* * *$ & 0,14 & $* *$ & 0,179 & $*$ \\
\hline Brand Trust & $\rightarrow$ & Brand Sensuality & H9 & 0,038 & NS & $-0,017$ & NS & 0,047 & NS \\
\hline Brand Intimacy & $\rightarrow$ & Brand Affect & $\mathrm{H} 10$ & $-0,056$ & NS & $-0,135$ & $* *$ & 0,049 & NS \\
\hline Brand Intimacy & $\rightarrow$ & Brand Sensuality & H11 & 0,026 & NS & 0,165 & $*$ & $-0,115$ & NS \\
\hline Brand Affect & $\rightarrow$ & Brand loyalty & $\mathrm{H} 12$ & 0,553 & $* * *$ & 0,575 & $* * *$ & 0,623 & $* * *$ \\
\hline Brand Sensuality & $\rightarrow$ & Brand loyalty & H13 & 0,177 & $*$ & 0,103 & NS & 0,195 & NS \\
\hline Brand Affect & $\rightarrow$ & $W O M$ & $\mathrm{H} 14$ & 0,641 & $* * *$ & 0,732 & $* * *$ & 0,662 & $* * *$ \\
\hline Brand Sensuality & $\rightarrow$ & $W O M$ & $\mathrm{H} 15$ & 0,101 & NS & $-0,014$ & NS & 0,115 & NS \\
\hline Brand Affect & $\rightarrow$ & Brand Love & H16 & 0,421 & $* * *$ & 0,488 & $* * *$ & 0,431 & $* * *$ \\
\hline Brand Sensuality & $\rightarrow$ & Brand Love & $\mathrm{H} 17$ & 0,319 & $* * *$ & 0,204 & NS & 0,407 & $* * *$ \\
\hline Brand Affect & $\rightarrow$ & Price Premium & $\mathrm{H} 18$ & 0,551 & $* * *$ & 0,669 & $* * *$ & 0,5 & $* * *$ \\
\hline Brand Sensuality & $\rightarrow$ & Price Premium & H19 & 0,055 & NS & $-0,153$ & NS & 0,235 & \\
\hline
\end{tabular}

Nota: *se $\mathrm{p}<0,1 ; * *$ se $\mathrm{p}<0,05 ; * * *$ se $\mathrm{p}<0,01$; NS se $\mathrm{p}>0,1$

Fonte: elaboração própria

A tabela 2 apresenta os resultados do modelo estrutural que testa as hipóteses propostas para essa investigação. Em primeiro lugar, importa destacar que os resultados apresentados corroboram a nossa hipótese central em que a "Brand Sensuality" tem um impacto direto e positivo sobre o "Brand Affect" (SRW=0,388; $\mathrm{p}<0,01$ ), suportando, assim, H1. Como referido anteriormente, a comunicação das marcas, que se baseiam na sensualidade para captar a atenção do público-alvo, traduzem uma mensagem que leva a estímulos ou desperta o prazer sensual na mente do consumidor (AMOR et al., 2013; FERNANDES, 2010). Na realidade, uma composição musical, um estímulo olfativo, uma variedade de texturas, experiências visuais, o logótipo e até a embalagem de determinado produto conduzem a associações agradáveis, produzindo o desejo 
sensual no consumidor. Quando as marcas conseguem apelar aos sentidos dos clientes, podem encontrar o caminho que leva a seus corações, desenvolvendo afetos duradouros entre marcas e consumidores (CHAUDHURI; HOLBROOK, 2001; ROBERTS, 2005). Salienta-se que essa hipótese foi observada, também, no gênero feminino ( $S R W=0,514, p<0,01)$, mas não no gênero masculino ( $S R W=0,223, p \geq 0,1$ ). Uma explicação possível sugerida pela literatura passa pelo fato de a sensualidade estar mais vinculada à mulher e não ao homem. Enquanto os homens estão associados às mais variadas formas de sucesso, $\mathrm{o}$ gênero feminino aparece valorizado pela beleza corporal. A mulher sempre foi mais ligada a uma maior carga afetiva e emocional do que o homem (SAMARÃO, 2007; VERÍSSIMO, 2005).

Relativamente ao "Brand Experience", verifica-se, na amostra global, que essa variável tem um impacto positivo quer no "Brand Affect" (H2: SRW=0,198; $p<0,1)$, quer na "Brand Sensuality" (H3: SRW=0,659; $\mathrm{p}<0,01$ ). A marca Nespresso conta com uma vasta experiência de marca no que se refere ao mercado do café. Com um design único, alta tecnologia, uma diversidade de gamas de produtos, verifica-se que cada detalhe foi concebido para que o cliente Nespresso usufrua de uma experiência inigualável (BREM; MAIER; WIMSCHNEIDER, 2016). Desse modo, as experiências positivas estimulam os sentidos humanos e permitem que o consumidor fique ligado a emoções, experiências corporais e cognições (DOLBEC; CHEBAT, 2013). A premissa principal da Nespresso passa por garantir ao consumidor a melhor experiência na degustação do café, oferecendo-lhe múltiplas formas de despertar os seus desejos e emoções, fruto da experiência de marca associada. Particularizando $\mathrm{H} 2$ por gênero, denotamos que essa hipótese não é corroborada no gênero feminino. Realça-se que a "Brand Experience" tem um impacto positivo sobre a "Brand Sensuality" (H3), uma vez que os aspetos sedutores provenientes da experiência levam aos consumidores a envolverem-se e a desenvolverem uma sensação ímpar e sem igual com essa marca (HOCH, 2002). Salien- ta-se o fato de que $\mathrm{H} 3$ é corroborada tanto em nível geral, como particularizada por gênero, estando assim, de acordo com a perspetiva de Hoch (2002) e Rodrigues (2014) que assumem que uma experiência positiva e fulcral para a criação da sensualidade da marca, ou seja, os aspetos sedutores provenientes de determinada experiência levam os consumidores a envolverem-se e a desenvolverem uma sensação ímpar com essa marca.

No que diz respeito ao "Hedonic Product", encontrou-se suporte estatístico, confirmando-se uma ligação positiva com o "Brand Affect" (H4) (SRW=0,352; $<<0,01)$. Uma vez que os produtos hedônicos estão diretamente associados a emoções e ao prazer, é expetável que seu consumo desperte fortes respostas emocionais e afetivas (CARROLL; AHUVIA, 2006). A marca Nespresso tem como objetivo o desenvolvimento de produtos inovadores, destacando-se no mercado como um segmento de alta qualidade de café, no qual desencadeia emoções e afetos a seus consumidores. Levando em consideração o gênero, evidencia-se que H4 também é suportada em ambos os gêneros. Analisando o impacto do "Hedonic Product" sobre a "Brand Sensuality", evidenciou-se que (H5) não é corroborada nesta investigação ( $S R W=0,152 ; p \geq 0,1$ ). Nesse caso, evidencia-se que a marca não desperta por meio dos seus produtos hedônicos a sensualidade da marca no comportamento do consumidor; contudo, particularizando por gênero, verificou-se que H5 é corroborada no gênero masculino (SRW=0,362; $<<0,01$ ), suportando a análise dos autores Esmailzadeh, Meral e Agilonu (2010). Nessa ótica, os produtos hedônicos transmitem ao consumidor uma experiência multidimensional, descritas em várias dimensões hedônicas, como fantasias, sentimentos e diversão (HIRSCHMAN; HOLBROOK, 1982).

Seguidamente, verificou-se que a ligação entre o "Brand identity Expressivenees" e o "Brand Affect" não foi corroborada (H6: SRW=$0,063 ; p>0,1)$. Quando isolada por gênero, verifica-se que H6 também não é corroborada nem no gênero feminino nem no gênero masculino, 
evidenciando-se, neste último caso, uma relação negativa, ou seja, uma relação contrária à hipótese que pressupõe uma relação positiva entre o "Brand identity Expressivenees" e o "Brand Affect". Igualmente ao caso anterior, presencia-se que a "Brand identity Expressivenees" também não tem impacto na "Brand Sensuality" (SRW=$0,022 ; \mathrm{p} \geq 0,1)$. Relativamente aos resultados associados à "Brand identity Expressivenees", estes não espelham os efeitos esperados. Talvez a escala utilizada no modelo não reflita, verdadeiramente, o significado pleno da "Brand identity Expressivenees".

Relativamente ao "Brand Trust", observa-se que essa variável tem um impacto positivo sobre o afeto à marca $(\mathrm{SRW}=0,145$; $\mathrm{p}<0,01)$. Dessa forma, pode afirmar-se que a H8 é corroborada. Se os consumidores tiverem plena confiança na marca, despertam uma maior carga emocional por ela, assim os afetos/ emoções têm um efeito primário sobre a confiança, evoluindo, subsequentemente, para uma resposta cognitiva (DUNN; SCHWEITZER, 2005; SCHOORMAN; MAYER; DAVIS, 2007). Analisando o impacto do "Brand Trust" na sensualidade da marca, verifica-se que H9 não é corroborada ( $S R W=0,038 ; p \geq 0,1$ ). Uma explicação possível poderá passar pelo fato de a marca Nespresso ter falhado (direta ou indiretamente) nos seus compromissos e valores; por exemplo, mau atendimento, um produto que foi uma desilusão, entre outros. Outra explicação poderá estar relacionada com o afastamento de George Clooney, em 2017, das campanhas publicitárias da Nespresso. Como se sabe, o simbolismo de George Clooney nas campanhas publicitárias da Nespresso traduzia os demais valores de luxo e status social inerentes à marca Nespresso. Durante vários anos, George Clooney foi embaixador da marca, tendo um papel representativo de aspetos como o luxo, o status social, a classe e a elegância inerente à própria marca. Após a sua saída, poderá ter existido uma quebra de confiança pela marca, uma vez que grande parte do público Nespresso via em George Clooney a cara da marca, sendo o elo representativo mais consistente e sólido da "Brand Sensuality" na marca Nespresso.
O impacto positivo da "Brand Intimacy" no "Brand Affect" (H10) não é corroborado nem em nível geral (SRW=-0,056; $p \geq 0,1$ ) nem na análise feita por género, evidenciando-se uma relação negativa entre a "Brand Intimacy" e o "Brand Affect" no gênero feminino. Por outro lado, analisando a relação entre a "Brand Intimacy" e a "Brand Sensuality", (H11) verificou-se, de igual forma, que essa hipótese não é corroborada nesta amostra em nível geral ( $\mathrm{SRW}=0,026 ; \mathrm{p}>0,1)$. Todavia, quando analisada em particular por gênero, demonstra-se que a H11 é corroborada pelo gênero feminino $(\mathrm{SRW}=0,165-; \mathrm{p}<0,1)$. A sensualidade da marca está, intrinsecamente, associada à intimidade, sendo recorrente a literatura descrever a importância das experiências íntimas entre marcas e consumidores para desencadear perceções associadas ao sex appel (GONÇALVES; NISHIDA, 2009; GOULD, 1992b; ROBERTS, 2005; SAMARÃO, 2007). Inúmeros estudos demonstram que experiências íntimas entre marca e consumidor aumentam o desejo sensual, por exemplo, cores agradáveis (BABIN; HARDESTY; SUTER, 2003), produtos perfumados (BONE; JANTRANIA, 1992) e músicas agradáveis nas lojas (YALCH; SPANGENBERG, 2000) aumentam as perceções e o desempenho das marcas, ativando, assim, o desejo sensual dos consumidores.

Passando agora para a discussão de hipóteses relativas aos consequentes dos dois construtos centrais, verificam-se os seguintes resultados: a variável "Brand Affect" tem um impacto positivo na "Brand Loyalty", (SRW=0,553; $<<0,01$ ), o que corrobora H12. Isso significa que a interação entre o consumidor e a marca desencadeia um leque diversificado de emoções positivas, induzindo por isso mesmo um alto nível de lealdade por parte do consumidor. Adicionalmente, constata-se que essa relação é corroborada em ambos os gêneros. Noutra ótica, verificou-se que a "Brand Sensuality" tem um impacto positivo sobre a "Brand Loyalty", o que suporta H13 ( $S R W=0,177 ; p<0,1)$. Na investigação dos autores Cho Fiore e Russell (2015) conclui-se que uma marca não deve canalizar os seus pro- 
dutos somente por meio de sites ou lojas para promover associações cognitivas e sensoriais. Dessa forma, para ampliar o relacionamento de lealdade entre consumidor e marca, devem promover-se programas de fidelidade e práticas de marketing interativo experiencial $(\mathrm{CHO}$; FIORE; RUSSELL, 2015). O marketing experiencial reforça várias dimensões (mistério, sensualidade e intimidade), consolidando, de forma positiva, as preferências, as perceções e o comportamento do consumidor (FENKO; SCHIFFERSTEIN; HEKKERT, 2010; FIORE; KIM, 2007; FIORE; YAH; YOH, 2000). A Nespresso, por meio de uma experiência sublime fruto do sistema único e exclusivo de cápsulas de café, juntamente com a tecnologia inovadora das máquinas, permite que a marca capte em simultâneo todos os sentidos e as perceções de forma a manter os consumidores seduzidos e leais pela experiência de consumo Nespresso.

Verifica-se, também, que o "Brand Affect" tem um impacto positivo sobre a variável "WOM", o que suporta H14 (SRW=0,641; $\mathrm{p}<0,01)$. Segundo Batra, Ahuvia e Bagozzi (2012) os consumidores que nutrem afetos por uma determinada marca estão mais abertos a falar dela, logo os efeitos das experiências afetivas são responsáveis pelos fenômenos do WOM (WESTBROOK, 1987; YI 1990). Os resultados obtidos corroboram H14, tanto na análise global, como na análise por gênero. Em relação à "Brand Sensuality" demonstra-se que não tem influência sobre o WOM nesta investigação, não suportando H15 (SRW=0,101; $\mathrm{p} \geq 0,1$ ), nem em nível global nem na análise por gênero.

Levando em consideração o "Brand Affect", presencia-se um impacto positivo para a formação do "Brand Love", suportando H16 $(\mathrm{SRW}=0,421 ; \mathrm{p}<0,01)$. O amor à marca está diretamente relacionado com as questões afetivas, ou seja, os consumidores relembram os bons momentos passados, o que é um excelente elo condutor entre as marcas e o sentimento de nostalgia. Como os afetos constroem o sentimento de amor; no contexto das marcas, o afeto positivo tem um impacto em nível da duração do relacionamento, bem como a for- mação do sentimento de "Brand Love" (BAIRRADA, 2015; BATRA; AHUVIA; BAGOZZI, 2012). Os resultados presentes corroboraram H16, tanto em nível da análise geral, como na análise feita por gênero. Observando o efeito da "Brand Sensuality", verifica-se que há um impacto positivo sobre o "Brand Love", suportando, dessa forma H17 (SRW=0,319; $<<0,01$ ). De acordo com a visão de Roberts (2005), corrobora-se que a sensualidade é uma das variáveis fundamentais para despertar o sentimento de amor pela marca; assim, quando as marcas conseguem apelar aos desejos dos clientes, podem encontrar o caminho que leva a seus corações. Quando particularizada por gênero, só se corrobora H17 no gênero masculino.

Por fim, constata-se que o "Brand Affect" tem um impacto positivo sobre o "Price Premium", suportando, assim, H18 (SRW=0,551; $\mathrm{p}<0,01)$. Logo, aumentando o relacionamento entre marca e consumidor fruto das perceções positivas e sinergias partilhadas, estes se tornam insensíveis ao fator preço, ou seja, quando um indivíduo está emocionalmente ligado à determinada marca, tende a aceitar um preço mais elevado para adquirir esse produto (MAMUM; RAHMAN; ROBEL, 2014; SUNG; KIM, 2010; THOMSON; MACINNIS; PARK, 2005). Evidencia-se, ainda, que H18 também é suportada em ambos os gêneros, demonstrando, assim, que os consumidores atribuem uma maior carga de tempo, energia e dinheiro pelas marcas que desenvolvem em si afetos, sendo elas elos emocionais entre marcas e consumidores (BATRA; AHUVIA; BAGOZZI, 2012). Propôs-se da mesma forma que os consumidores estão dispostos a pagar mais por determinada marca que desperta atrações e desejos sensuais. Nessa amostra, evidencia-se que a "Brand Sensuality" tem apenas um impacto positivo sobre o "Price Premium" perante o gênero masculino, o que suporta H19 (SRW $=0,235 ; \mathrm{p}<0,1$ ), não sendo H19 corroborada em nível geral nem no género feminino.

\section{DISCUSSÃO E CONCLUSÕES}

Esta investigação teve como principal 
objetivo compreender a "Brand Sensuality" e "Brand Affect", os seus antecedentes e consequentes. Desse modo, procurou-se perceber como os construtos de sensualidade e afeto à marca são percecionados pelos consumidores, mais especificamente pelos consumidores da marca Nespresso.

Os principais resultados alcançados mostram, em nível geral, que a "Brand Experience" é um bom antecedente da "Brand Sensuality" e no que toca a seus consequentes, verificou-se seu impacto no "Brand Love" e "Brand Loyalty".

Em relação ao "Brand Affect", verificou-se uma maior consistência nos resultados. Relativamente a seus antecedentes, pode-se evidenciar o papel do "Brand Experience", "Hedonic Product" e "Brand Trust". No espetro dos consequentes, evidenciam-se a relevância de todos os construtos considerados neste trabalho de investigação: "Brand Loyalty", "WOM", "Brand Love" e "Price Premium", comprovando, assim, uma forte ligação entre o "Brand Affect" e todos os seus consequentes, sustentando a importância dessa variável no comportamento do consumidor e na gestão de marcas.

\section{CONTRIBUIÇÕES}

\subsection{CONTRIBUIÇÕES TEÓRICAS}

Esta pesquisa apresenta um modelo de investigação subdividido em duas grandes partes centrais, uma referente à "Brand Sensuality" e outra referente ao "Brand Affect".

Dado que a "Brand Sensuality" é um tema pouco referenciado na literatura, esta investigação apresenta-se como inovadora. No campo teórico, o estudo do comportamento do consumidor evidencia os demais temas que podem interagir com a "Brand Sensaulity" e o "Brand Affect".

A originalidade desta investigação passa, assim, pelos seguintes aspetos: em primeira instância, destaca-se pela diferenciação clara da "Brand Sensuality" e "Brand Sense"; por vezes, muito confundida por parte dos acadêmicos. Em segundo lugar, comprovou-se a hipótese central em que a "Brand Sensuality" tem um impacto positivo sobre o "Brand Affect"; por último, estudou-se, simultaneamente, os efeitos do "Brand Affect" e da "Brand Sensuality" de forma a clarificar alguns dos seus antecedentes e consequentes.

Esta investigação consolidou o fato de que a "Brand Experience" se trata de uma variável de extrema importância quando a construção da "Brand Sensuality". No que respeita aos consequentes, destacou-se a importância da "Brand Sensuality" sobre o "Brand Love" e "Brand Loyalty".

No que refere ao "Brand Affect", reforçou-se o papel da "Brand Experience", o "Hedonic Product" e "Brand Trust" como antecedentes fundamentais para atingir o afeto à marca. Por último, reforçaram-se todos os consequentes relativos ao "Brand Affect" sendo eles: "Brand Loyalty". "WOM", "Brand Love" e "Price Premium".

\subsection{CONTRIBUIÇÕES PRÁTICAS PA- RA AS EMPRESAS}

Por meio do estudo desses dois construtos, "Brand Affect" e "Brand Sensuality", as empresas podem melhorar as suas estratégias de branding reforçando consideravelmente a relação entre marcas e consumidores. Dessa forma, importa destacar que as estratégias de marketing com sucesso passam por vincular às marcas uma enorme carga emocional (THOMPSON; RINDFLEISCH; ARSEL, 2006).

A comunicação das marcas que se baseiam na sensualidade para captar a atenção do público-alvo traduz uma mensagem que leva a estímulos ou desperta o prazer sensual na mente do consumidor, por meio da conjugação de experiências sensoriais únicas que atingem as perceções dos consumidores ("Brand Experience").

Neste estudo, reforça-se a perspetiva de Roberts (2005), em que a sensualidade é uma das variáveis fundamentais para despertar o sentimento de amor pela marca, em nível prático, que a experiência reforça várias dimensões (mistério, sensualidade e intimidade) realçando, de 
forma positiva, a lealdade dos consumidores.

O presente trabalho pretende contribuir com alguns insights essenciais para que as marcas cativem, cada vez mais, os consumidores.

Por meio do conhecimento aprofundado dos construtos "Brand Affect" e "Brand Sensuality", as empresas podem construir estratégias de fidelização de clientes mais eficientes indo ao encontro dos sentimentos dos consumidores. As análises dessa investigação permitem chegar a conclusões acrescidas sobre as marcas, o mercado, os consumidores, bem como outros fenômenos associados ao marketing. Sendo a "Brand Sensuality" analisada, pela primeira vez, como variável central. Esta investigação oferece aos profissionais da área do marketing novas perspetivas.

O conhecimento da "Brand Sensuality" permite que as empresas despertem, junto a seus consumidores, prazeres e apelos sensuais por meio do contacto com a marca, criando um sentimento de excitação e partilha de sinergias ímpares entre marca e consumidor, podendo até, futuramente, suscitar amor pela marca (CHO; FIORE; RUSSELL, 2015).

A criação de afeto à marca por parte das organizações influencia os consumidores a nutrirem bons sentimentos e recordações, permitindo, assim, um elo verdadeiro ente marcas e consumidores. Atualmente, o mercado mostra-se cada vez mais competitivo e em constante evolução, pois estratégias com base nesses dois construtos desempenham um papel diferenciador e inovador na gestão de marcas, ou seja, com implantação de estratégias desse tipo, as empresas podem ganhar, cada vez mais, o aspeto emocional dos consumidores e, consequentemente, aumentarem as suas margens de lucro.

\subsection{LIMITAÇÕES E RECOMENDA- ÇÕES PARA PESQUISAS FUTURAS}

Esta investigação testou algumas variáveis enquanto antecedentes e consequentes da "Brand Sensuality" e "Brand Affect", apresentando contribuições tanto do ponto de vista teórico, como prático; contudo, apresenta algu- mas limitações, deixando pistas para futuras investigações. Sugere-se a repartição do modelo em modelos menores, introduzindo novas variáveis para apurar o conhecimento da "Brand Sensuality". A variável que se sugere ser implementada em um modelo futuro refere-se à compra impulsiva, sendo possível que seja um consequente forte da "Brand Sensuality" (CHO; FIORE; RUSSELL, 2015; THOMSON; MACINNIS; PARK, 2005).

As conclusões retiradas deste estudo foram amplas a todas as comunidades de língua portuguesa; todavia, não podem ser generalizadas para uma cultura, ou seja, os aspetos culturais podem divergir de cultura para cultura, em que a perceção e o conceito de afeto e/ou sensualidade poderá atingir um significado mais amplo ou mais restrito.

\section{REFERÊNCIAS}

AAKER, J. Dimensions of Brand Personality. Journal of Marketing Research, v. 34, n. 3, p. 347-356, 1997.

AMOR, N. E. et al. Print Advertising and Female Sensuality in a North African Context: The Mediating Role of Visual Mental Imagery. International Journal of Business and Social Science, v. 4, n. 13, p. 50-64, 2013.

BABIN, B. J.; HARDESTY, D. M.; SUTER, T. A. Color and shopping intentions: The intervening effect of price fairness and perceived affect. Journal of Business Research, v. 56, n. 7, p. 541-551, 2003. https://doi.org/10.1016/ S0148-2963(01)00246-6

BAGOZZI, R. P.; GOPINATH, M.; NYER, P. U. The Role of Emotions in Economic. Journal of the Academy of Marketing Science, v. 27, n. 2, p. 184-206, 1999. https://doi.org/10.1001/ jama.1953.03690150021006

BAIRRADA, C. Determinantes e consequências do amor pela marca: um estudo empírico. 2015. 221 f. Tese (Doutorado em Gestão de 
Empresas) - Universidade de Coimbra, Coimbra, 2015.

BATRA, R.; AHUVIA, A.; BAGOZZI, R. P. Brand Love. Journal of Marketing, v. 76, p. 1-16, 2012.

BONE, P. F.; JANTRANIA, S. Olfaction as a cue for product quality. Marketing Letters, v. 3, n. 3, p. 289-296, 1992. https://doi. org/10.1007/BF00994136

BRAKUS, J. J.; SCHMITT, B. H.; ZARANTONELLO, L. Brand experience: what is it? how is it measured? does it affect loyalty? Journal of Marketing, v. 73, n. 3, p. 52-68, 2009. https://doi.org/10.1509/jmkg.73.3.52

BREM, A.; MAIER, M.; WIMSCHNEIDER, C. Competitive advantage through innovation: the case of Nespresso. European Journal of Innovation Management, v. 19, n. 1, p. 133148, 2016. https://doi.org/10.1108/EJIM-052014-0055

CARPENTER, J. M.; FAIRHURST, A. Consumer shopping value, satisfaction, and loyalty for retail apparel brands. Journal of Fashion Marketing and Management: An International Journal, v. 9, n. 3, p. 256-269, 2005. https://doi.org/10.1108/13612020510610408

CARROLL, B. A.; AHUVIA, A. C. Some antecedents and outcomes of brand love. Marketing Letters, v. 17, n. 2, p. 79-89, 2006. https:// doi.org/10.1007/s11002-006-4219-2

CHAUDHURI, A.; HOLBROOK, M. B. The Chain of Effects from Brand Trust and Brand Affect to Brand Performance: The Role of Brand Loyalty. Journal of Marketing, v. 65, n. 2, p. 81-93, 2001. https://doi.org/10.1509/ jmkg.65.2.81.18255

CHERNATONY, L.; RILEY, F. Defining A "Brand": Beyond The Literature With Experts' Interpretations. Journal of Marketing Mana- gement, v. 14, n. 5, p. 417-443, 1998. https:// doi.org/10.1362/026725798784867798

CHI, H. K.; YEH, H. R.; CHIOU, C. Y. The effect of Brand Affect on Female Cosmetic User Brand Loyalty in Taiwan. The Journal of American Academy of Business, v. 14, n. 1, p. 230236, 2009. https://doi.org/10.2307/3151897

CHO, E.; FIORE, A.; RUSSELL, D. Validation of a Fashion Brand Image Scale Capturing Cognitive, Sensory, and Affective Associations: Testing Its Role in an Extended Brand Equity Model. Psychology and Marketing, v. 32, n. 1, p. 28-48, 2015. https://doi.org/10.1002/mar

DHAR, R.; WERTENBROCH, K. Consumer Choice Between Hedonic and Utilitarian Goods. Journal of Marketing Research, v. 37, n. 1, p. 60-71, 2000. https://doi.org/10.1509/ jmkr.37.1.60.18718

DICK, A. S.; BASU, K. Customer Loyalty: Toward an Integrated Conceptual Framework. Journal of the Academy of Marketing Science, v. 22, n. 2, p. 99-113, 1994. https://doi. org/10.1177/0092070394222001

DOLBEC, P. Y.; CHEBAT, J. C. The Impact of a Flagship vs. a Brand Store on Brand Attitude, Brand Attachment and Brand Equity. Journal of Retailing, v. 89, n. 4, p. 460-466, 2013. https://doi.org/10.1016/j.jretai.2013.06.003

DUNN, J. R.; SCHWEITZER, M. E. Feeling and believing: The influence of emotion on trust. Journal of Personality and Social Psychology, v. 88, n. 5, p. 736-748, 2005. https:// doi.org/10.1037/0022-3514.88.5.736

EREVELLES, S. The Role of Affect in Marketing. Journal of Business Research, v. 42, n. 3, p. 199-215, 1998. https://doi.org/10.1016/ S0148-2963(97)00118-5

ESMAILZADEH, E.; MERAL, S.; AGILONU, B. The Secret of Love: A Case of Love- 
marks. Evaluation, p. 1-115, 2010.

FENKO, A.; SCHIFFERSTEIN, H. N. J.; HEKKERT, P. Shifts in sensory dominance between various stages of user-product interactions. Applied Ergonomics, v. 41, n. 1, p. 34-40, 2010. https://doi.org/10.1016/j.apergo.2009.03.007

FERNANDES, P. R. A imagem da Mulher: Uma análise da representação do corpo feminino nas fotografias publicitárias contemporâneas. Intercom - Sociedade Brasileira de Estudos Interdisciplinares Da Comunicação, p. 1-14, 2010. https://doi.org/10.7764/cdi.36.647

FEYBESSE, C.; NETO, F.; HATFIELD, E. Adaptação da escala de amor apaixonado na população portuguesa. Psicologia, Educação e Cultura, v. 15, n. 1, p. 161-180, 2011.

FIORE, A. M.; KIM, J. An integrative framework capturing experiential and utilitarian shopping experience. International Journal of Retail \& Distribution Management, v. 35, n. 6, p. 421-442, 2007. https://doi. org/10.1108/09590550710750313

FIORE, A. M.; YAH, X.; YOH, E. Effects of a product display and environmental fragrancing on approach responses and pleasurable experiences. Psychology and Marketing, v. 17, n. 1, p. 27-54, 2000. https://doi.org/10.1002/ (SICI) 1520-6793(200001) 17:1<27::AID-MAR3>3.0.CO;2-C

FOG, K.; BUDTZ, C.; YAKABOYLU, B. Storytelling: Branding in practice. South Asian Journal of Management, v. 12, n. 3, p. 92-95, 2005. https://doi.org/10.1007/978-3540-88349-4

FOURNIER, S. Consumers and Their Brands: Developing Relationship Theory in Consumer Research. Journal of Consumer Research, v. 24, n. 4, p. 343-353, 1998.
GONÇALVES, E.; NISHIDA, N. Publicidade e ética : um estudo da construção da imagem da mulher. Revista Comunicação, Mídia e Consumo, v. 6 , n. 17, p. 49-72, 2009. Retrieved from http://revistacmc.espm.br/index.php/ revistacmc/article/view/166/167

GOULD, S. J. The Production, Marketing, and Consumption of Sexually Explicit Material in Our Sexually Conflicted Society: A Public Policy Dilemma. Journal of Public Policy \& Marketing, v. 11, n. 2, p. 135-148, 1992b. https://doi.org/10.1177/074391569201100213

HAIR, J. et al. Multivariate data analysis. Harlow: [s.n.], 2014.

HERSKOVITZ, S.; CRYSTAL, M.. The essential brand persona: Storytelling and branding. Journal of Business Strategy, v. 31, n. 3, p. 21-28, 2010. https://doi. org/10.1108/02756661011036673

HIRSCHMAN, E.; HOLBROOK, M. Hedonic Consumption: Emerging Concepts, Methods and Propositions. Journal of Marketing, v. 46, n. 3, p. 92-101, 1982. https://doi. org/10.1002/9781118785317.weom090127

HOCH, S. J. Product Experience Is Seductive. Journal of Consumer Research, v. 29, n. 3, p. 448-454, 2002. https://doi.org/10.1086/344422

KELLER, K. L. Conceptualizing, Measuring, and Managing Customer-Based Brand Equity. Journal of Marketing, v. 57, n. 1, p. 1-22, 1993.

KRISHNA, A. An integrative review of sensory marketing: Engaging the senses to affect perception, judgment and behavior. Journal of Consumer Psychology, v. 22, n. 3, p. 332-351, 2012. https://doi.org/10.1016/j. jcps.2011.08.003

LIN, M. Q.; LEE, B. C. Y. the Influence of Website Environment on Brand Loyalty: Brand 
Trust and Brand Affect As Mediators. International Journal of Electronic Business Management, v. 10, n. 4, p. 308-321, 2012. https:// doi.org/10.1509/jmkg.65.2.81.18255

LINDSTROM, M. Brand Sense: a marca multissensorial. [S.l.]: Bookman, 2007.

LIPOVETSKY, G. Sedução, publicidade e pós-modernidade. Revista FAMECOS, v. 12, p. 7-13, 2000.

LISBOA, J. V.; AUGUSTO, M. G.; FERREIRA, P. L. Estatística aplicada à gestão. Porto: [s.n.], 2012.

MAMUM, A. A.; RAHMAN, M. K.; ROBEL, S. A Critical Review of Consumers' Sensitivity to Price: Managerial and Theoretical Issues. Journal of International Business and Economics, v. 2, n. 2, p. 1-9, 2014.

MATZLER, K.; BIDMON, S.; GRABNER-KRÄUTER, S. Individual determinants of brand affect: The role of the personality traits of extraversion and openness to experience. Journal of Product and Brand Management, v. 15, n. 7, p. 427-434, 2006. https://doi. org/10.1108/10610420610712801

MCALEXANDER, J. H.; SCHOUTEN, J. W.; KOENIG, H. F. Marketplace Communities A Broader View of Brand Community. Journal of Marketing, v. 66, p. 38-54, 2002.

MORGAN, R. M.; HUNT, S. D. The Commitment-trust theory of relationship marketing. Journal of Marketing, v. 58, n. 3, p. 20-38, 1994.

PEREIRA, M.; COELHO, A.; BAIRRADA, C. O Impacto dos Sentidos na Atitude face à Marca: Um estudo do grupo Inditex. International Journal of Marketing, Communication and New Media, v. 5, n. 7, p. 67-84, 2016.

PLUMMER, J. How personality makes a dif- ference. Journal of Advertising Research, v. 40, n. 6, p. 79-83, 2000.

ROBERTS, K. Lovemarks: The future beyond brands. New York: Powerhouse Books, 2005. Retrieved from https://www.kobo.com/us/en/ ebook/lovemarks

RODRIGUES, C. Brand sensuality and consumer-based brand equity. 2014. $149 \mathrm{f}$. Tese (Doutorado em Ciências Empresariais) - Universidade do Porto, Porto, 2014. Disponível em: https://repositorio-aberto.up.pt/bitstream/10216/76153/2/31376.pdf. Acesso em: 1 ago. 2018.

ROOK, D. W. The Ritual Dimension of Consumer Behavior. Journal of Consumer Research, v. 12, n. 3, p. 251-264, 1985. https://doi. org/10.1086/208514

RUSSELL, J. A. Core Affect and the Psychological Construction of Emotion. American Psychological Association, Inc, v. 110, n. 1, p. 145-172, 2003. https://doi.org/10.1037/ 0033-295X.110.1.145

RUSSELL, J. A.; BARRETT, L. F. Russell-Barrett-1999. Journal of Personality and Social Psychology, v. 76, n. 5, 1999. https://doi. org/10.1037//0022-3514.76.5.805

SABAT, R. Pedagogia cultural, gênero e sexualidade. Revista Estudos Feministas, v. 9, n. 1, p. 9-21, 2001. https://doi.org/10.1590/s0104026x2001000100002

SAMARÃO, L. A representação do corpo feminino na mídia. Revista ContemporâNea, v. 8, p. 45-57, 2007.

SAMETZ, R.; MAYDONEY, A. Storytelling through design. Design Management Journal, v. 14, n. 4, p. 18-34, 2003. https://doi.org/10.1111/j.1948-7169.2003.tb00347.x

SARKAR, A.; PONNAM, A. Understanding 
and measuring romantic brand love. Journal of Customer Behaviour, v. 11, n. 4, p. 324347, 2012. https://doi.org/10.1362/14753921 2X13546197909985

SCHMITT, B. Experiential Marketing. Journal of Marketing Management, v. 15, n. $1 / 3$, p. 53-67, 1999. https://doi. org/10.4324/9781315175614-7

SCHOORMAN, D.; MAYER, R. C.; DAVIS, J. $H$. An integrative model of organizational trust: past, present, and future. Academy of Management Review, v. 32, n. 2, p. 344-354, 2007. https://doi.org/10.1145/2435349.2435386

SHOEMAKER, S.; LEWIS, R. C. Customer loyalty: the future of hospitality marketing. International Journal of Hospitality Management, v. 18, n. 4, p. 345-370, 1999. https://doi. org/10.1016/S0278-4319(99)00042-0

SOUZA, I.; LEÃO, A. Movimento sensual: um estudo da narrativa mitológica na publicidade de uma marca de moda praia. Organizações \& Sociedade, v. 20,n. 67,p.623-649,2013. https:// doi.org/10.1590/s1984-92302013000400004

SUNG, Y.; KIM, J. Effects of Brand Personality on Brand Trust and Brand Affect. Psychology \& Marketing, v. 27, n. 7, p. 639-661, 2010. https://doi.org/10.1002/mar

TEIXEIRA, R. B. Discurso publicitário e a pedagogia do gênero: representações do feminino. Revista Comunicação, Mídia e Consumo, v. 6, n. 17, p. $37-48,2009$.

TELLEGEN, A.; WATSON, D.; CLARK, L. A. On the dimensional and hierarchical structure of affect. American Psychological Society, v. 10, n. 4, p. 297-303, 1999.

THOMPSON, C.; RINDFLEISCH, A.; ARSEL, Z. Emotional Branding and the Strategic Value of the Doppelgänger Brand Image. Journal of Marketing, v. 70, p. 50-64, 2006.
Retrieved from http://journals.ama.org/doi/ $\mathrm{abs} / 10.1509 / \mathrm{jm} .09 .0339$

THOMSON, M.; MACINNIS, D. J.; PARK, C. W. The ties that bind: measuring the strength of the ties that bind thomson, macinnis, park consumers' emotional attachments to brands. Journal of Consumer Psychology, v. 15 , n. 1, p. 77-91, 2005. https://doi. org/10.1108/13612021211265872

VALDEZ, P.; MEHRABIAN, A. Effects of Color on Emotions. Journal of Experimental Psychology: General, v. 123, n. 4, p. 394-409, 1994. https://doi.org/10.1037/00963445.123.4.394

VERÍSSIMO, J. A mulher "objecto" na publicidade. Livro de Actas Do $4^{\circ}$ Congresso Da Associação Portuguesa de Ciências Da Comunicação. 2005. p. 1-13. Disponível em: http:// www.bocc.ubi.pt/pag/verissimo-jorge-mulher-objecto-publicidade.pdf

WESTBROOK, R. Affective Product / Consumption-B Responses and. Journal of Marketing Research, v. 24, n. 3, p. 258-270, 1987.

WORTHINGTON, S.; RUSSELL-BENNETT, R.; HÄRTEL, C. A tri-dimensional approach for auditing brand loyalty. Journal of Brand Management, v. 17, n. 4, p. 243-253, 2009. https://doi.org/10.1057/bm.2009.24

XIE, Y.; BATRA, R.; PENG, S. An Extended Model of Preference Formation Between Global and Local Brands: The Roles of Identity Expressiveness, Trust, and Affect. Journal of International Marketing, v. 23, n. 1, p. 50-71, 2015. https://doi.org/10.1509/jim.14.0009

YALCH, R. F.; SPANGENBERG, E. R. The effects of music in a retail setting on real and perceived shopping times. Journal of Business Research, v. 49, n. 2, p. 139-147, 2000. https:// doi.org/10.1016/S0148-2963(99)00003-X 
YI, Y. Cognitive and affective priming effects of the context for print advertisements. Journal of Advertising, v. 19, n. 2, p. 40-48, 1990. https://doi.org/10.1080/00913367.1990.10673 186

ZAJONC, R. B. Feeling and Thinking Preferences Need No Inferences. Zhonghua Yi Shi Za Zhi, v. 35, n. 2, p. 151-175, 1980. https:// doi.org/10.1037/0003-066X.35.2.151 